\title{
Immunophenotypic characterisation of morphologically diagnosed cases of Acute Myeloid Leukaemia (AML)
}

\author{
Maria Basharat ${ }^{1}$, Saleem Ahmed Khan ${ }^{2}$, \\ Nasir ud din ${ }^{3}$, Dawood Ahmed ${ }^{4}$
}

\begin{abstract}
Objective: To determine immunophenotypic pattern in newly diagnosed cases of acute myeloid leukaemia by flow cytometry and its correlation with morphological findings.

Methods: This study was conducted at Haematology (Pathology) department, Army Medical College, in collaboration with Immunology Department Armed Forces Institute of Pathology, Rawalpindi from 16 November 2016 to 16 November 2017. One hundred and six patients of both genders and all age groups diagnosed as acute myeloid leukaemia were included in the study. Demographic data was noted. Complete blood counts, bone marrow examination and cytochemical stains were carried out and evaluated microscopically for blast percentage and morphology. Immunophenotyping was performed by flow cytometry using standard panel on peripheral blood or bone marrow samples. The surface and cytoplasmic antigens of interest were analysed and correlated with morphological findings.

Results: The most commonly expressed antigens were CD13, CD33, CD45 and HLA-DR. Almost all blasts expressed CD45 with no remarkable difference among the subtypes of AML. The mean positivity for CD13 among all AML subtypes was $57 \%$ and for CD33 was $67 \%$. Aberrant expression of CD7 and CD19 were expressed in $26.4 \%$ and $1.1 \%$ of all cases respectively. There was concordance rate of $90 \%$ between morphology and FCM in our study.

Conclusion: Flow cytometric analysis of acute leukaemia done by a combination of patterns and intensity of antigen expression improves diagnostic yield in AML. CD13, CD33 and CD45 are the most frequently expressed antigens in AML. Our findings suggest a $90 \%$ concordance between morphology and flow cytometry. It is pertinent to conclude that flow cytometry results interpreted with morphology are complementary.
\end{abstract}

KEYWORDS: Immunophenotyping, Acute myeloid leukemia (AML), Aberrant phenotype in AML, Flowcytometry.

doi: https://doi.org/10.12669/pjms.35.2.614

How to cite this:

Basharat M, Khan SA, Nasir ud Din, Ahmed D. Immunophenotypic characterisation of morphologically diagnosed cases of Acute Myeloid Leukaemia (AML). Pak J Med Sci. 2019;35(2):470-476. doi: https://doi.org/10.12669/pjms.35.2.614

This is an Open Access article distributed under the terms of the Creative Commons Attribution License (http://creativecommons.org/licenses/by/3.0), which permits unrestricted use, distribution, and reproduction in any medium, provided the original work is properly cited.

Correspondence:

Dr. Maria Basharat, MBBS.

Pathology Department,

Army Medical College,

National University of Medical Sciences,

Post Code: 46000

Islamabad, Pakistan.

E-mail: mariabash86@yahoo.com

* Received for Publication:

* $1^{\text {st }}$ Revision Received:

* $2^{\text {nd }}$ Revision Received:

* $3^{\text {rd }}$ Revision Received:

* Final Revision Accepted:
March 26, 2018

April 24, 2018

June 22, 2018

December 14, 2018

January 20, 2019

\section{INTRODUCTION}

Acute Myeloid Leukemia (AML) is a clonal disorder of haemopoietic stem cells. It is characterised by inhibition of differentiation resulting in accumulation of cells at various stages of incomplete maturation. There is a decreased production of mature haemopoietic elements. ${ }^{1}$ This relatively common haematological malignancy comprises $80 \%$ of acute leukaemias in adults and $20 \%$ in children. ${ }^{2}$ Being the second most common type of leukaemia in the United States, 
studies have shown AML to be the commonest leukaemia in Pakistan. ${ }^{3}$

Diagnostic methods for acute leukemias include immature cell count, morphology, cytochemistry, immunophenotyping, cytogenetics and histochemistry in correlation with clinical features. All these diagnostic methods are complementary. Cell morphology remains the basic diagnostic tool to assess the number and morphology of blasts. Immunophenotyping by flow cytometry is a powerful adjuvant tool in delineating cell surface and cytoplasmic markers in AML. The expression of characteristic myeloid lineage markers CD13, CD33 and CD117 allows the distinction of AML from other types of leukaemias. ${ }^{4}$

In the diagnosis of acute leukemia concordance between experienced observers increases from 70 to 99\% when morphologic criteria are supplemented by cytochemical and immunophenotypic information. ${ }^{5} \quad$ Immunophenotyping plays an important role when morphological interpretation is difficult. The main advantage of immunophenotyping is identification of particular leukaemia subtype that cannot be identified by morphological criteria alone. Immunophenotyping of peripheral blood and bone marrow in leukaemia determines the decision making for a specific therapeutic regimen and is a practical prognostic indicator. ${ }^{6}$ The diagnosis and management of acute leukaemia depends primarily on the detection, identification and characterization of leukemic cells. ${ }^{7}$

Immunophenotypic markers shown in various studies implicating adverse outcomes are CD7, CD9, CD11b, CD13, CD14, CD33, CD34, CD56 and terminal deoxynucleotidyl transferase. ${ }^{8}$ Regardless of the increasing importance of molecular and genetic features in the sub classification of acute leukemias, morphology and immunophenotyping remain the primary modalities by which leukemias are evaluated. ${ }^{9}$

Aberrant phenotype is expression of lymphoidassociated markers in myeloblasts or that of myeloid associated markers in lymphoblasts. ${ }^{7}$ Aberrant immunophenotypic expression has been used to predict treatment outcome. ${ }^{10}$ The aim of our study was to determine immunophenotypic patterns of de novo AML cases by flow cytometry and to correlate with morphological, French American British (FAB) classification. Rationale of this study was to characterize immunophenotypic pattern of newly diagnosed patients of AML by flow cytometry (FCM) so that the diagnosis of AML can be improved and its correlation with morphological findings.

\section{METHODS}

This descriptive cross sectional study was carried out in Haematology Department Army Medical College in collaboration with Haematology and Immunology department of Armed forces institute of pathology, Rawalpindi from 16 November 2016 to 16 November 2017. The sampling technique used was non probability convenient sampling Approval was taken from ethical review board and institutional review board. A total of 106 patients of all ages and both genders who were newly diagnosed with AML were included. Relapsed cases of AML and those evolving from myelodysplasia (MDS) or receiving treatment for AML were excluded.

The samples were analysed for blast percentage and morphology on leishman stained peripheral blood and bone marrow smears followed by cytochemicalstains (Sudan Black B). Immunophenotyping was done using three colour flow cytometer. This included samples of peripheral blood or bone marrow aspirate from patients of De novo AML.

Sample Collection and Preparation: Two and a half $\mathrm{ml}$ of venous blood in Ethylene Diamine Tetra Acetic Acid (EDTA) tube was collected for Complete Blood Count (CBC) and peripheral blood film examination, under aseptic conditions. Bone marrow aspiration was done after written informed consent, following standard guidelines. Complete blood counts were generated using automated haematology analyzer Sysmex KX-21. Leishman stained smears from peripheral blood and bone marrow aspirates were examined under microscope for morphology and percentage of blasts. ${ }^{11}$

Lysing of Whole Blood and Combination of B.M Aspiration, Monoclonal Antibody: Labelling of each test tube (Falcon, BD) was done properly and placed in sequence. Ten $\mu \mathrm{l}$ of monoclonal antibody was added into each tube. $50 \mu$ l of whole blood / diluted bone marrow in each tube was added, thoroughly mixed and incubated in dark for 30 minutes at room temperature. Fluorescent activated cell sorter lysing solution (FACSLyse) in distilled water was prepared in 1:10 dilution. In each test tube $2 \mathrm{ml}$ of diluted FACSLyse was added and incubated in dark for five minutes at room temperature. It was centrifuged at $300 \mathrm{~g}$ for five minutes at room temperature. The supernatant was discarded and the remaining $50 \mu$ fluid was 
Immunophenotyping and Aberrant phenotype in AML

Table-I: Distribution of positive results of different markers among different AML subtypes.

\begin{tabular}{ccccccccc}
\hline $\begin{array}{c}\text { AML Subtype } \\
(F C M)\end{array}$ & $N$ & Weak CD45 & HLA-DR & CD 13 & CD 33 & CD 34 & CD 14 & CD7 \\
\hline AML M0 & 4 & $82.0 \%$ & $75 \%$ & $64.00 \%$ & $49.75 \%$ & $73.75 \%$ & $0.0 \%$ & $0.0 \%$ \\
AML M1 & 15 & $79 \%$ & $70 \%$ & $56.00 \%$ & $72.53 \%$ & $45.33 \%$ & $0.0 \%$ & $27.06 \%$ \\
AML M2 & 49 & $71.24 \%$ & $57 \%$ & $50.53 \%$ & $60.18 \%$ & $44.81 \%$ & $0.0 \%$ & $13 \%$ \\
AML M3 & 19 & $82 \%$ & $12 \%$ & $64.73 \%$ & $72.52 \%$ & $11.68 \%$ & $0.0 \%$ & $1.48 \%$ \\
AML M4 & 16 & $52 \%$ & $62.8 \%$ & 71.812 & $86.81 \%$ & $38.06 \%$ & $44.12 \%$ & 0.00 \\
AML M6 & 3 & $29.33 \%$ & $28 \%$ & $22.00 \%$ & $30.00 \%$ & $1.33 \%$ & $0.0 \%$ & $15.0 \%$ \\
\hline Total & 106 & $70.42 \%$ & $51.49 \%$ & $56.71 \%$ & $66.91 \%$ & 37.7925 & $6.72 \%$ & $10.33 \%$ \\
\hline
\end{tabular}

shaken for resuspension of cells. In each tube $2 \mathrm{ml}$ of phosphate buffered saline (PBS) was added. Centrifuge at $300 \mathrm{~g}$ for five minutes and supernatant was discarded. The remaining fluid was shaken and $0.5 \mathrm{ml}$ of $3.3 \%$ formalin was added to each test tube. The samples were kept at $4^{\circ} \mathrm{C}$ till analysis on flow cytometer.

Immunophenotyping by flow cytometry: Flow cytometric analysis was made by a top FACScan flow cytometer (Becton \& Dickenson). The flourochromes FITC, PE and PerCP were used. The primary panel of monoclonal antibodies (mAbs) used were CD45 (for blast identification), CD13, CD33, CD34, HLADR and for AML M4/M5 CD14, for AML M6 antiglycophorin A and for AML-M7, CD61, CD41 and CD42 were used. The extended panel included CD117 and Anti-MPO was used in cases where the morphology suggested AML-M $\mathrm{M}_{0}$ and where results of primary panel were inconclusive. The populations were considered positive if at least $20 \%$ of cells within gate showed the expression of a particular antigen. For cytoplasmic antigen expression, the threshold was $10 \%$.

Quality control: Isotype control (antimouse IgG1FITC/Ig2aPE) is used as negative control (should not react with human blood/bone marrow cells). If it happens, the test/reagent should be rechecked.

\section{RESULTS}

Data was analyzed using SPSS version 21. Frequencies and percentages were calculated for variables like gender. For quantitative data analysis, mean along with standard deviation were used to assess the values for monoclonal antibodies Correlation tests were applied using Pearson correlation coefficient. The age of the patients at the time of diagnosis ranged from one year to 80 years (mean age $=31.81 \pm 19.972$ years). Males constituted $58.5 \%$ and females were $41.50 \%$. The male to female ratio was 1.4:1.

The mean haemoglobin levels were $8.25 \pm 2.16 \mathrm{~g} /$ $\mathrm{dl}$. The lowest haemoglobin level was $3 \mathrm{~g} / \mathrm{dl}$ seen in AML-M2 and highest levels in AML-M3 of $13.50 \mathrm{~g} /$ $\mathrm{dl}$. The mean TLC was $57.46 \pm 79.39$, the lowest being $0.99 \times 10^{9} / \mathrm{L}$ and highest being $456.77 \times 10^{9} / \mathrm{L}$ both extremes seen in AML-M1.The mean of platelet count was $58.23 \pm 81.29 \times 10^{9} / \mathrm{L}$. The highest count was $1000 \times 10^{9} / \mathrm{L}$ seen in AML-M2 and lowest being $659 \times 10^{9}$ seen in AML-M1.

The least number of blasts were 22(22\%) seen in AML-M2 and AML-M6 and highest abnormal promyelocytes with reniform nuclei were $98 \%$, found in AML-M3. Out of 106 cases analyzed, AML-M2 was the most frequent subtype constituting $49(47.2 \%)$ cases followed by AML-M3 $(\mathrm{n}=18,17 \%$,). The least common being AML-M6 $(n=3,2.8 \%)$ as shown in Fig.1. All cases expressed

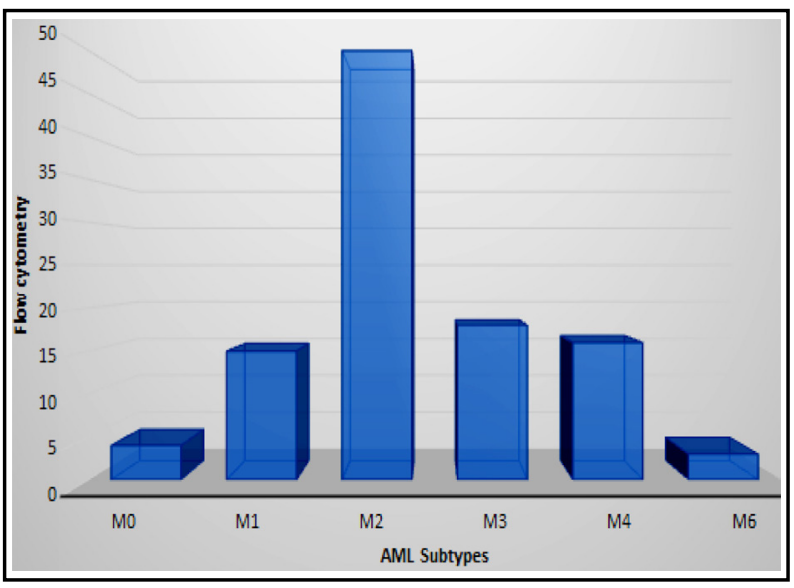

Fig.1: Frequency of subtypes of AML by immunophenotyping. 
CD45 with mean positivity of $70.42 \% \pm 23.74$ and no significant variations among the subtypes as shown in Table-I. HLA-DR was expressed in $51.49 \% \pm$ 33.92 of all AML cases. The strongest positivity was seen in $A M L-M_{O}(75 \% \pm 33.42)$ and weakest positivity in AML-M3 $(12 \% \pm 23.84)$. CD34 showed mean positivity of $38 \% \pm 30.84$ among all AML subtypes, strongest being in AML- $\mathrm{M}_{\mathrm{O}}(74 \% \pm 21.79)$ and weakest seen in AML-M6 $(1.33 \% \pm 2.31)$. In our study there were 18 cases ( $\mathrm{n}=106)$ of AML-M3 of

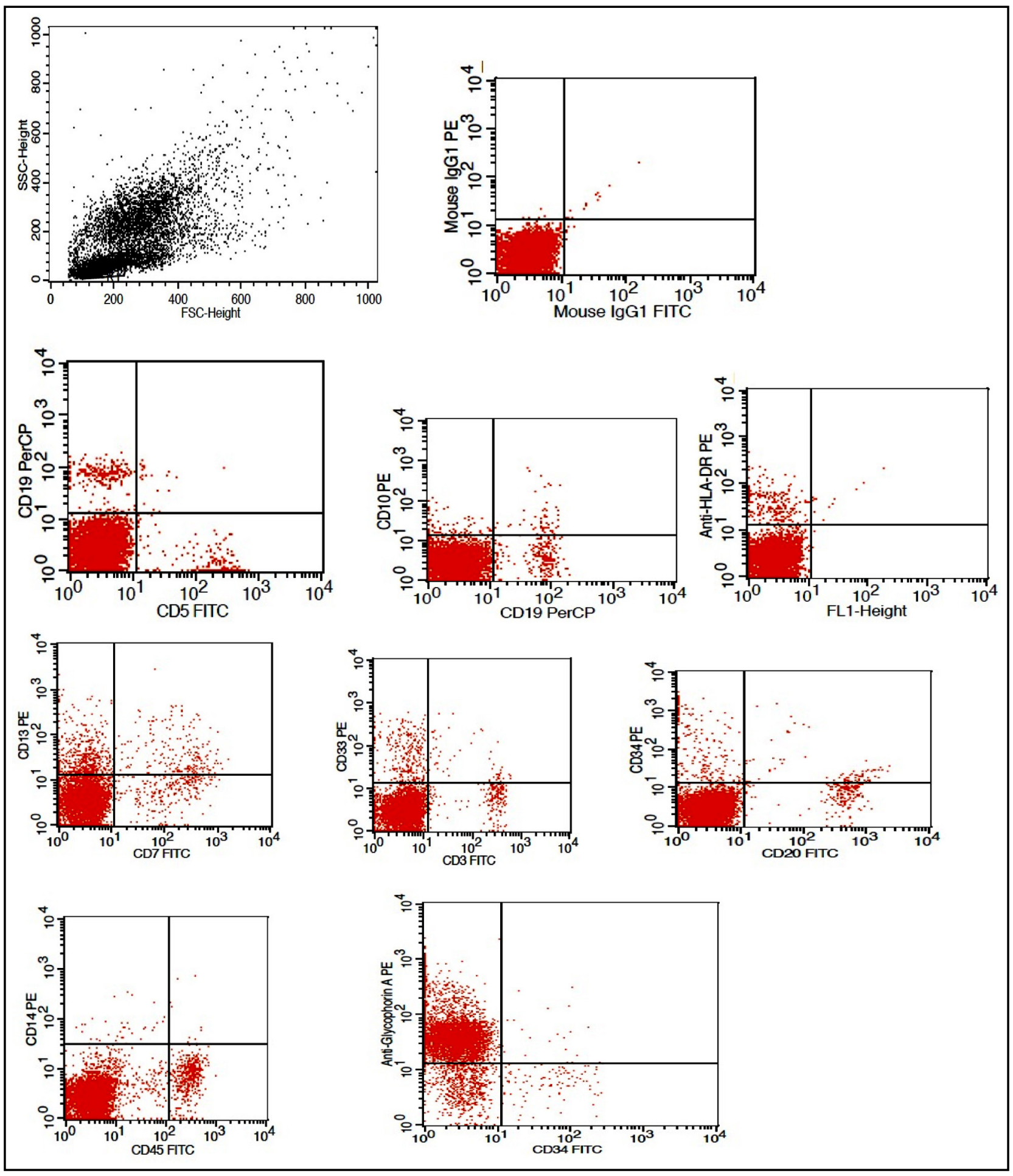

Fig.2: Flow cytometric findings in a case of AML-M6. 
which only 1 case of AML-M3 showed positivity for HLA-DR and 3 cases $(n=18)$ were positive for CD34, however none of them were positive for both CD34 and HLA-DR. CD13 was studied among the blast population with mean positivity of $57 \%$ \pm 27.53 among all subtypes with strongest positivity in AML-M4 $(72 \% \pm 20.01)$ and weakest in AML-M6 $(22 \% \pm 1.73)$.

CD33 showed mean positivity of $67 \% \pm 27.56$, strongest being in AML-M4 $(87 \% \pm 9.02)$ weakest in AML-M6 $(30 \% \pm 11.14)$.CD13 and CD33 were also studied for correlation using Pearson correlation and was found to be statistically significant among all subtypes. The strongest correlation being in AML-M6 $(r=0.962)$ and weakest being in $\mathrm{AML}^{-M_{0}}$ $(\mathrm{r}=0.717)$.

The concordance rate between morphology and FCM is seen in $102(90 \%)$ cases with confidence interval of $95 \%$ in our study. While partial discordance was seen in $4(3.77 \%)$ cases where lineage was correctly identified with difference in defining subtypes only.

The aberrant expression of CD7 showed positivity in $28(26.4 \%)$ cases, most frequently expressed in AML-M2 and CD19 was expressed in 1(1.1\%) case. The results of correlational analysis showed that CD 13, CD 34, CD7 and CD14 had a significant correlation with immunophenotyping $(r=-.321$, .360 .297 and -.585 respectively) and insignificant correlation with CD33, CD117 and CD19 (r=.111, .141 and .031 respectively).

\section{DISCUSSION}

Immunophenotyping has a recognised role in the diagnosis and classification of acute leukaemia. ${ }^{12}$ AML has an age adjusted incidence of 3.7/100,000 per annum in US with highest incidence in $7^{\text {th }} \mathrm{dec}-$ ade. ${ }^{3}$ AML can occur at any age group but the incidence increases with age. ${ }^{2}$ The mean age in our study was 32 years similar to Harani et al. ; However, in some other studies mean age was more than our study ranging from 35 to 47 years. ${ }^{(2,3,13-15)}$

Childhood AML comprised $27.3 \%$ in our study while adult AML comprised $72 \%$ which was slightly different from Gosh study. (24\%,76\% respectively). ${ }^{16}$ Conversely, in Ahmad et al., paediatric cases were $20 \%$ and adults $86.4 \%,{ }^{3}$ There is a predilection for men with AML, 4.8 versus 3.3 new cases whereas in ALL, there is no gender variance (1.9 new cases in men and 1.5 in women) ${ }^{17}$ In our study the male to female ratio was 1.2:1 indicating a slight male predominance is similar to some other international studies. ${ }^{2,5,13,16,18}$ In Patel et al., the male to female ratio was 1:1.1. ${ }^{19}$

In our study AML-M2 (47.2\%) was the most frequent subtype similar to Gosh study (AML-M2=34\%). ${ }^{16}$ Conversely, in few other international studies ${ }^{3,5,14}$, other AML subtypes predominated.

CD7, a T-cell antigen known to show aberrant expression was most commonly expressed in our study $(26.4 \%)$ followed by CD19 (1.2\%). The same trend was observed in most of the international studies where CD7 was most commonly expressed aberrant antigen followed by CD19. ${ }^{2,8,14,16,20}$ CD7 expression in AML is correlated with lower incidence of complete remission. ${ }^{2}$ According to Belurkar et al., expression of lymphoid associated antigens except CD7, on AML blasts lack prognostic significance and CD7 + AML is a particular subset but in general, it may not represent a biologically distinct form of leukemia since these cases have similar clinical features and a comparable response to therapy. ${ }^{21}$

The combined use of CD34 and HLA-DR was more helpful in distinguishing Acute Promyelocytic Leukemia (APL) from non-Acute Promyelocytic Leukemia (non-APL) none of the eighteen cases of APL were positive for both CD34 and HLA-DR whereas $64.77 \%$ of non-APL $(83 \%)$ were positive for both CD34 and HLA-DR. While out of eighty-eight non-APL, two $(2.27 \%)$ cases were negative for both HLA-DR and CD34 as compared to $77.87 \%$ of APL. Thus the negativity for these two antigens doesn't refer to APL diagnosis exclusively.

For more than 20 years, the FAB classification for acute leukemia has been the major system of classification. This classification enabled the diagnosis of a variety of morphologic and cytochemical subtypes of acute leukemia through a structured criterion. However, studies are indicative of failure of most of categories of FAB system in delineating significant disease groups based on morphology and cytochemistry in terms of survival of patients. ${ }^{21}$ In our study we analyzed 106 cases of AML by FCM and compared them with their morphological (FAB) diagnosis as well as frequency of their immunological patterns. There was concordance rate of $90 \%$ between morphology and FCM of AML while partial discordance rate was $3.77 \%$.

In case 1, morphological diagnosis was that of AML-M3 while on FCM; HLA-DR and CD34 
were positive and was reported as AML-M2. In case 2, morphology suggested AML-M5 but FCM showed negative expression for HLA-DR, CD34, and CD14, reported as AML-M3. Morphological diagnosis of case 3 was AML-M6 but FCM showed negativity for anti-glycophorin A and was reported as AML-M2. Morphology in case 4 was suggestive of AML-M2. However, on FCM positivity of CD14 suggested AML-M4. This discordance was resolved on cytogenetic and molecular analysis. Morphological analysis along with cytochemical stain (SBB) rendered the diagnosis in $>80 \%$ of our AML cases. Similar study was carried out by Berlukar et al. ${ }^{21}$, where the complete concordance rate was $58 \%$, partial concordance $22 \%$. In Kheiri et al., the concordance rate was $77.4 \%$ with $89.2 \%$ of the myeloid leukemias showing agreement between morphology and $\mathrm{FCM}^{22}$, in Mhawech et al., the concordance rate was $80.32 \%{ }^{23}$ The difference between our study and the above mentioned studies is that we included only AML while these studies compared the morphology and FCM of acute leukemias that is both ALL and AML which explains high concordance rate in our study. Morphology and immunophenotyping complement each other mainly because they have as common objects malignant cell phenotype as a whole (morphology, i.e. surface and intracellular marker expression). Conversely, morphology is burdened by a high degree of subjectivity and flow cytometry techniques have not consensus standard protocols yet. That is why correlation of results provided by the two techniques is still absolutely necessary. ${ }^{24}$

Limitations of the study: One of the limitations was that this study was conducted on a relatively small sample size. the specific markers for AML were used but there was limited use of markers like $\mathrm{MPO}$ and CD117. Both these markers are one of the most sensitive markers of myeloid lineage. These were used in only a few cases. Moreover this study would improve if parallel use of molecular analysis was done. However, there were only very few cases with molecular analysis.

\section{CONCLUSION}

It is concluded that immunophenotyping is an essential tool in diagnosis and classification of AML. Our findings suggest a $90 \%$ concordance between morphology and flow cytometry However, it depends upon the availability of facility for broad panel of primary and secondary markers, which is available only in specialized centres. Interpretation of immunophenotyping should be done in close correlation with morphological findings as both these complement each other. It is need of the hour to extend facility of immunophenotyping at regional levels in the country so that access to these sophisticated facilities is available to patients of leukemias to avoid long distance travel by members of this diseased community.

\section{RECOMMENDATIONS}

Our findings suggest complementary use of immunophenotyping with morphology improves outcome in the diagnosis of acute leukemias. Further research with use of extended panel of monoclonal antibodies is recommended. Additionally, development of complementary diagnostic techniques such as cytogenetics and molecular analysis should be developed for more precise diagnosis of AML.

Conflict of Interest: None.

Funding Disclosure: None.

Grant Support \& Financial Disclosures: None.

\section{REFERENCES}

1. Ferrara F, Schiffer CA. Acute myeloid leukaemia in adults. Lancet. 2013;381(9865):484-495.

2. Osman IM, Humeida AA, Eltayeb O, Abdelrhman I, Elhadi TA. Flowcytometric Immunophenotypic characterization of Acute Myeloid Leukemia (AML) in Sudan. Int J Hematol Dis. 2015;2(1):10-17. doi: 10.12691/ijhd-2-1-3.

3. Ahmad SQ, Yusuf R, Burney S. Frequency of various types of leukaemias diagnosed at PAF hospital mianwali. Pak Arm Forces Med J. 2015;65(4):474-477.

4. Ambayya A. recent advances in diagnosis of acute myeloid leukaemia: A review. Asian J Multidis Stud. 2013;1(5):145-146.

5. Harani MS, Adil SN, Shaikh MU, Kakepoto GN, Khurshid $\mathrm{M}$. Frequency of fab subtypes in acute myeloid leukemia patients at Aga Khan University Hospital Karachi. J Ayub Med Coll Abbottabad. 2005;17(1):26-29

6. Tsegaye A. Diagnostic Utility Of Immunophenotyping By Flow Cytometry For Diagnosis And Classification Of Acute Leukemias In Tikur Anbessa Specialized Hospital, Addis Ababa, Ethiopia (Doctoral Dissertation, Addis Ababa University).

7. Jahedi M, Shamsasenjan K, Sanaat Z, Aliparasti M, Almasi $S$, Mohamadian $\mathrm{M}$, et al. Aberrant Phenotype in Iranian Patients with Acute Myeloid Leukemia. Adv Pharm Bull. 2014;4(1):43-47. doi:10.5681/apb.2014.007.

8. Webber BA, Cushing MM, Li S. Prognostic significance of flow cytometric immunophenotyping in acute myeloid leukemia. Int J Clin Experimen Pathol. 2008;1(2):124-133.

9. Peters JM, Ansari MQ. Multiparameter flow cytometry in the diagnosis and management of acute leukemia. Arch Pathol Lab Med. 2011;135(1):44-54. 
10. Ossenkoppele GJ, Loosdrecht AA, Schuurhuis GJ. Review of the relevance of aberrant antigen expression by flow cytometry in myeloid. Brit J Haematol. 2011;153(4):421-436. doi: 10.1111/j.1365-2141.2011.08595.x.

11. Bain BJ, Lewis SM. Preparation and staining methods for blood and bone marrow films. In: Bain BJ, Bates I, Laffan MA, Lewis SM, editors. Dacie Lewis Pract Haematol. 11 ed. London: Churchill Livingstone; 2012: 116.

12. Venkateswaran SP, Jojo A, Vidhyadharan G, Unni M. A Clinicopathological Correlation of Acute Leukaemias in relation to Immunophenotyping and Cytogenetics. International Journal of Collaborative Research on Internal Medicine and Public Health (IJCRIMPH). 2012;4:1713-1736.

13. Khalid A, Zahid M, Rehman A, Ahmad ZU, Qazi S, Aziz Z, et al. Clinicoepidemiological features of adult leukemias in Pakistan. J Pak Med Assoc. 1997;47(4):119-122.

14. Salem DA, El-Aziz SMA. Flowcytometric immunophenotypic profile of acute leukemia: mansoura experience. Ind J Hematol Blood Transfus. 2012;28(2):89-96.

15. Abdulateef $\mathrm{N}$, Ismail $\mathrm{MM}$, Aljedani $\mathrm{H}$. Clinical significance of co-expression of aberrant antigens in acute leukemia: a retrospective cohort study in Makah Al Mukaramah, Saudi Arabia. Asian Pac J Cancer Prev. 2014;15(1):221-227.

16. Ghosh S, Shinde SC, Kumaran GS, Sapre RS, Dhond SR, Badrinath $\mathrm{Y}$, et al. Haematologic and immunophenotypic profile of acute myeloid leukemia: An experience of Tata Memorial Hospital. Ind J Cancer. 2003;40(2):71-76.

17. Fiegl M. (2016) Epidemiology, pathogenesis, and etiology of acute leukemia. In: Hiddemann W. (eds) Handbook of Acute Leukemia. Adis, Cham , p-4.

18. Baviskar JB. Incidence of acute and chronic leukemias in rural area at tertiary care teaching hospital: a five years of study. Ind J Pathol Oncol. 2016;3(4):710-713.

19. Patel K, Lotodo T, Njuguna F, Emonyi W, Mining S, Buziba $\mathrm{N}$, et al. Use of Flow Cytometry Immunophenotyping for Diagnosis of Acute Leukemia at Moi Teaching and Referral Hospital, Eldoret, Kenya. Am Scient Res J Engin Technol Sci. 2015;13(1):72-80.
20. Jha R, Grover G, Bose P. Lymphoid associated antigen expression in new cases of Acute Myeloid Leukemia. J Pathol Nepal. 2013;3(6):487-490.

21. Belurkar S, Mantravadi H, Manohar C, Kurien A. Correlation of morphologic and cytochemical diagnosis with flowcytometric analysis in acute leukemia. J Cancr Res Ther. 2013;9(1):71. doi: 10.4103/0973-1482.110378.

22. Kheiri SA, MacKerrell T, Bonagura VR, Fuchs A, Billett HH. Flow cytometry with or without cytochemistry for the diagnosis of acute leukemias? Cytometry. 1998;34(2):82-86.

23. Mhawech P, Buffone GJ, Khan SP, Gresik MV. Cytochemical staining and cytometry methods applied to the diagnosis of acute leukemia in the pediatric population: an assessment of relative usefulness. J Pediat Hematol Oncol. 2001;23(2):89-92.

24. Selicean EC, Patiu M, Cucuianu A, Dima D, Dobreanu M. Correlation of cytomorphology with flowcytometric immunophenotyping in acute myeloid leukemia. Romanian Rev Lab Med. 2013;21(3):333-341. doi: 10.2478/ rrlm-2013-0028.

\section{Authors' Contribution:}

MB: Did data collection, statistical analysis \& and manuscript writing.

SAK: Conceived, designed, review and final approval of manuscript.

ND: Did statistical analysis, review \& editing of manuscript.

DA: Did statistical analysis \& review of manuscript.
Authors:

1. Maria Basharat, MBBS.

2. Saleem Ahmed Khan, MBBS, MCPS, FCPS, FRCP, PhD.

3. Nasir ud din MBBS, FCPS.

4. Dawood Ahmed MBBS, FCPS.

1-4: Pathology Department, Army Medical College, National University of Medical Sciences, Islamabad, Pakistan. 